\title{
Studies on fish diversity concerning Hydro-ecology of Kangsabasti river in Purulia district, West Bengal, India
}

Manab Kumar Saha

\author{
Department of Zoology, \\ Ramananda Centenary College, \\ Laulara, PURULIA-723151 (W.B.), INDIA. \\ Email-manabsaha5@gmail.com
}

Received : $18.10 .2020 ;$ Accepted : 16.11 .2020

\begin{abstract}
Fish diversity depends both on various physicochemical parameters and the biological components of the riverine ecosystem. During the study period from January 2017 to December 2019 the highest fish diversity and density were observed in post-monsoon and lowest in pre-monsoon season in the Kangsabati River, Purulia District of West Bengal. Twenty five fish species, associated with 19 genera, 10 families and 5 orders have been identified. It was recorded that the Cyprinidae was the predominant family, which represented $56 \%$ of the entire fish catch.
\end{abstract}

Figures : $01 \quad$ References : 26

Tables : 06

KEY WORDS : Ecological integrity, Fish diversity, Kangsabati River, Physicochemical parameters, West Bengal.

\section{Introduction}

In aquatic ecosystem, the water serves as the lifeline for all the aquatic organisms ${ }^{21}$. The fishes are considered as indicator species to check the aquatic health (Physiochemical property of water) of a particular aquatic ecosystem ${ }^{13}$. Over the past few decades, changes in ecological and environmental conditions show a negative impact on river diversity. Many fish species have become endangered ${ }^{8,11}$. For that reason, the conservation of river fish diversity has become more essential and gets the maximum priority ${ }^{23}$. There was no previous record regarding the icthyofaunal diversity of river Kangsabati in Purulia district. For this study, several attempts were made to collect, classify and identify fish of river Kangsabati to document the fish diversity, abundance and richness concerning different water parameters viz. Temperature, Conductivity, Transparency, dissolved oxygen, $\mathrm{pH}$, salinity, conductivity and zooplankton density, etc $4,6,9,11$.

\section{Study Area:}

The River Kangsabati is also known as the 'Kasai I Kansai' originates at Jabor Pahar (Gayalikocha, $\left.23^{\circ} 16^{\prime} 49^{\prime \prime} \mathrm{N} 86^{\circ} 08^{\prime} 23^{\prime \prime E}\right)$ from the Chhota Nagpur plateau in Purulia district, state of West Bengal, India.

\section{Materials and Methods}

During the survey period, sampling was done bimonthly viz. pre-monsoon (March-May), monsoon (June-September), and post-monsoon (OctoberNovember) and, winter (December-February). The average annual 'species-wise fish' catch value was determined by adding the bimonthly average value for each season ${ }^{8}$. From each site (sub site) data were collected regarding fish fauna and physicochemical parameters of river water (Table-5). Fishes were collected from each sampling station and different fishing gears viz. scoop net, drag net, cast net, gill net, hooks and lines were used for sampling ${ }^{5}$. The fish samples were photographed immediately before release them into the river at same place. Only few 'indicative specimens' from captured fishes were fixed in $8 \%$ formalin solution for further identification ${ }^{8}$. The fish specimens were identified with the help of key ${ }^{18,19,25}$. The zooplankton was identified (Rotifera, Cladocera, Copepoda, Protozoa) with the help of a microscope and compared with the identification 
TABLE-1. 'Sampling sites and their physical attributes parameters at Kangsabati River, Purulia district'.

\begin{tabular}{|c|c|c|c|c|c|}
\hline S. No. & Sampling sites & Position & Land use patterns & \multicolumn{2}{|c|}{ Distance (Km.) } \\
\hline Site I & Haranama river bank & $\begin{array}{l}23^{\circ} 18^{\prime} 39^{\prime \prime} \mathrm{N} \\
86^{\circ} 17^{\prime} 57^{\prime \prime} \mathrm{E}\end{array}$ & Rural, Agriculture & 21.39 & \multirow{5}{*}{104.75} \\
\hline Site II & Anai river bank & $\begin{array}{l}23^{\circ} 16^{\prime} 20^{\prime \prime} \mathrm{N} \\
86^{\circ} 26^{\prime} 36^{\prime \prime} \mathrm{E}\end{array}$ & $\begin{array}{l}\text { Rural, Agriculture, } \\
\text { domestic sewage }\end{array}$ & 21.72 & \\
\hline Site III & Kaira/Bansa bridge & $\begin{array}{l}23^{\circ} 10^{\prime} 17^{\prime \prime} \mathrm{N} \\
86^{\circ} 36^{\prime} 6^{\prime \prime} \mathrm{E}\end{array}$ & $\begin{array}{l}\text { Rural, Agriculture, domestic } \\
\text { sewage, temple in the river } \\
\text { bank, sand mining. }\end{array}$ & 26.85 & \\
\hline Site IV & Pairachali bridge & $\begin{array}{l}23^{\circ} 4{ }^{\prime} 52^{\prime \prime} \mathrm{N} \\
86^{\circ} 42^{\prime} 32^{\prime \prime} \mathrm{E}\end{array}$ & $\begin{array}{l}\text { Rural, Agriculture, domestic } \\
\text { sewage, temple in the river } \\
\text { bank, sand mining. }\end{array}$ & 19.83 & \\
\hline Site V & Darasol river bank & $\begin{array}{l}23^{\circ} 02^{\prime} 29^{\prime \prime} \mathrm{N} \\
86^{\circ} 43^{\prime} 22^{\prime \prime} \mathrm{E}\end{array}$ & Rural, Agriculture & 14.96 & \\
\hline
\end{tabular}

keys $^{3}$

Fish diversity index (relative abundance, Species Richness, Shannon-wiener index, Evenness index, Simpson's dominance index), the Pearson Correlation matrix $(r)$ between the physicochemical parameter and icthyofaunal diversity was done using Stat Plus software ${ }^{12}$ to observe the correlation among them.

\section{Result}

During the study period a total of 1655 fishes were recorded. According to the record (Table-2) fish specimens were distributed in 19 genera, 10 families and 5 orders. The statistical analysis shows that order Cyprinifomes $(60 \%)$ was highest in value, where-as Perciformes (24\%) and Siluriformes (8\%) subsequently occupy the next position (Table-2). Most of the individual fish species of the river showed the relatively rare abundance distribution, but nine species among them showed good relative abundance viz., Puntius ticto (15.4\%), Amblypharyngodon mola (15.34\%), Osteobrama cotio cotio (12.99\%), Salmophasia bacaila (12.8\%), P. sophore $(8.58 \%)$, Mystus tengara (4.71\%), Chanda nama (4.59\%), Labeo bata $(4.47 \%)$, C. ranga (3.26\%) were recorded at all the sites. It was also observed that the family Cyprinidae $(56 \%)$ was very common and dominant, followed by Ambassidae and Osphronemidae of the total fish catch.

According to the trophic utilization by fishes, herbivores (56\%) showed the highest number followed by omnivores (32\%) and carnivores (12\%). The present study did not record any exotic species in entire stretch.
As per IUCN Red list $80 \%$ of the fish species of Kangsabati River belong to least concern category ${ }^{24}$, while $12 \%$ is noted as near threaten. The Shannon-wiener index ( $\left.\mathrm{H}^{\prime}\right)$ within the 5 sites of the river varied from 2.52-2.62; Evenness index $(\mathrm{J})$ varied from 0.44-0.45; Simpson's dominance index (D) varied from 0.09-0.10; Simpson's diversity index (1-D) varied from 0.9-0.91 (Table 3).

Table 2. IUCN Red list- DD: Data Deficient, LC: Least Concern, VU: Vulnerable, NE: Not Evaluated, EN: Endangered, NT: Near Threatened; Population Trend-DE: Decreasing, UN: Unknown, ST: Stable; HEV: Herbivore, OMV: Omnivore, CAV: Carnivore.

Pearson's $r$ correlation between different parameters (Biotic and abiotic factors) is presented in (Table-4). To analyse the outcome of the Pearson's $r$ correlation, it was clear that they were either positively or negatively correlated between them. A strong and significant correlation was reported among the concentration of Dissolved Oxygen and the zooplankton density $(r=0.592, p<0.05)$, a very strong positive significant correlation $(r=0.747, p<0.05)$ was observed between the zooplankton density and the level of $\mathrm{pH}$. And two more relations were reported very significant, which were Salinity and the zooplankton density $(r=$ $0.681, p<0.05)$, the zooplankton density and Species Richness of fishes $(r=0.666, p<0.05)$. The other Positive correlations were also found between $\mathrm{pH}$ level Dissolved Oxygen $(r=0.974)$ along the river ${ }^{18}$.

On the other hand, significant negative correlations were found between the Transparency and Water 


\begin{tabular}{|c|c|c|c|c|c|c|c|c|c|c|c|c|c|c|}
\hline & $\begin{array}{l}\bar{\pi} \\
\text { 0 }\end{array}$ & ஜ & 岕 & $\hat{N}$ & ฉి & 今 & 8 & 음 & $F$ & N & ల్ల & $\stackrel{\infty}{N}$ & مొم & $\underset{F}{\stackrel{F}{ }}$ \\
\hline & $>$ & $\delta$ & 字 & 18 & d & $\delta$ & $\delta$ & $\delta$ & $\delta$ & $\underset{\ulcorner}{ }$ & ஜ & 广 & $\stackrel{\infty}{q}$ & 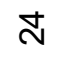 \\
\hline$\stackrel{\bar{\pi}}{0}$ & $\geq$ & ชิ & g & J & ஜ & $\delta$ & $\delta$ & ชิ & ชิ & $\underset{\nabla}{ }$ & $\hat{o}$ & $\stackrel{\mathscr{Y}}{\mathcal{H}}$ & 요 & $\stackrel{\sim}{N}$ \\
\hline 崖 & $\equiv$ & $\widetilde{\delta}$ & 5 & $1 \%$ & \& & $\delta$ & $\delta$ & $\widetilde{\delta}$ & $\widetilde{\delta}$ & $\stackrel{6}{\sim}$ & $\varnothing$ & $\stackrel{\mathscr{Y}}{\mathcal{H}}$ & 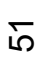 & $\mathbb{N}$ \\
\hline & $=$ & $\delta$ & กิ & $\mathscr{\varnothing}$ & $\mathscr{\varnothing}$ & ণิ & ชิ & ชิ & ஜூ & $\stackrel{10}{\leftarrow}$ & ஜ & $\forall$ & กิ & $\bar{m}$ \\
\hline & - & ऽ & 范 & 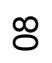 & オ & ช & $\bar{\delta}$ & $\varnothing$ & $\varnothing$ & $\stackrel{\varphi}{\circ}$ & 8 & $\mathscr{q}$ & 范 & శ్ \\
\hline 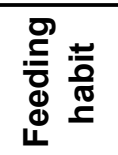 & & $\sum_{0}$ & 离 & 岸 & $\sum_{0}^{\gtrless}$ & 岌 & 岌 & 岸 & 岌 & 岌 & 岌 & 岌 & 岌 & 岌 \\
\hline $\begin{array}{l}0 \\
\frac{0}{ \pm} \\
\frac{\pi}{5} \\
0 \\
0 \\
0\end{array}$ & & 㟔 & $\bar{\omega}$ & $\bar{\omega}$ & Z & $\mathfrak{\omega}$ & Z & 㟔 & $\grave{\omega}$ & $\grave{\omega}$ & $\mathfrak{\omega}$ & 㟔 & 㟔 & $\mathfrak{\omega}$ \\
\hline 글 & & U & U & U & U & U & 々 & U & U & U & U & U & U & U \\
\hline 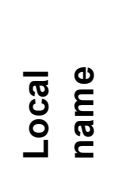 & & $\frac{\frac{\pi}{\sqrt{*}}}{\underline{\underline{T}}}$ & $\begin{array}{l}\frac{\pi}{0} \\
\frac{0}{3} \\
\frac{0}{2}\end{array}$ & $\underset{0}{\stackrel{\pi}{\mathbb{T}}}$ & 氶 & 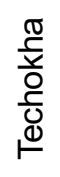 & 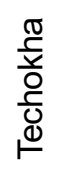 & 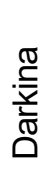 & 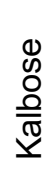 & $\begin{array}{l}\frac{\pi}{\pi} \\
\infty \\
0\end{array}$ & $\overline{\underline{\alpha}}$ & 吾 & 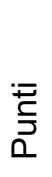 & 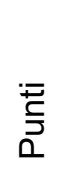 \\
\hline 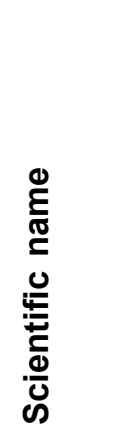 & & 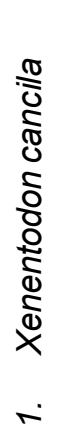 & 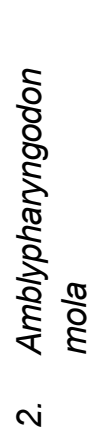 & 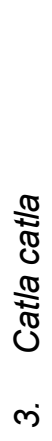 & 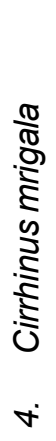 & 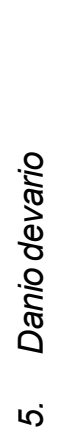 & $\begin{array}{l}\frac{0}{0} \\
\frac{0}{0} \\
\frac{0}{\overparen{D}} \\
0\end{array}$ & 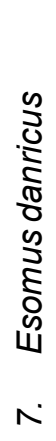 & 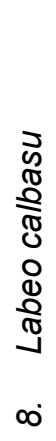 & 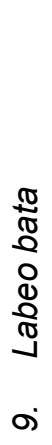 & 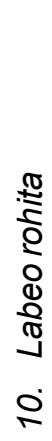 & 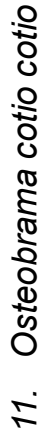 & 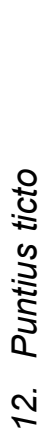 & 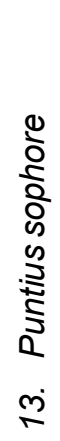 \\
\hline 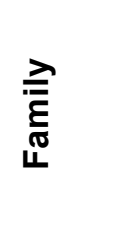 & & $\begin{array}{l}\frac{0}{\pi} \\
\frac{\pi}{0} \\
\frac{0}{\overline{0}} \\
\frac{0}{0}\end{array}$ & 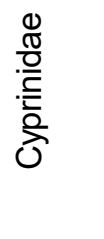 & & & & & & & & & & & \\
\hline के & & 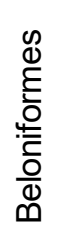 & 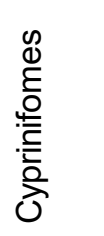 & & & & & & & & & & & \\
\hline
\end{tabular}




\begin{tabular}{|c|c|c|c|c|c|c|c|c|c|c|c|c|}
\hline 밈 & $\stackrel{N}{N}$ & $\stackrel{\infty}{\square}$ & $\approx$ & 芯 & $\stackrel{0}{R}$ & $\stackrel{\infty}{\circ}$ & $\stackrel{\circ}{\leftarrow}$ & g & ిె & $\stackrel{\infty}{\sim}$ & ஜூ & $\begin{array}{l}\stackrel{L}{0} \\
\stackrel{0}{0}\end{array}$ \\
\hline 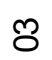 & $\infty$ & 8 & $\widetilde{O}$ & $\infty$ & $\simeq$ & $\widetilde{O}$ & $\delta$ & $\varnothing$ & 8 & $\stackrel{m}{\longrightarrow}$ & 8 & $\bar{S}$ \\
\hline ஜ & F & $\widetilde{O}$ & $\widetilde{O}$ & 8 & $\underset{\nabla}{ }$ & $\delta$ & ஜ & 8 & 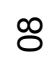 & $\stackrel{20}{2}$ & 8 & $\underset{\Gamma}{\bar{m}}$ \\
\hline ষ & F & \& & 8 & $F$ & $\stackrel{\varphi}{\oplus}$ & $\delta$ & ஜ & $F$ & 8 & $\stackrel{\circ}{\circ}$ & 8 & ల్ల \\
\hline ষ & 寸 & J & J & $\cong$ & $\stackrel{\varphi}{\sim}$ & $\widetilde{ఠ}$ & ๑ & $\simeq$ & ৪ & $\stackrel{\circ}{\circ}$ & 5 & 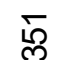 \\
\hline 뉴 & $\varphi$ & ஜ & 8 & $\underset{\square}{\mp}$ & $\stackrel{\infty}{\sim}$ & $\widetilde{\sigma}$ & ஜ & 8 & 응 & $\stackrel{\infty}{\circ}$ & ช & :্ల \\
\hline 岌 & $\underset{⿱ 亠 䒑}{\ddot{I}}$ & $\sum_{0}$ & $\sum_{O}$ & $\sum_{0}$ & $\sum_{0}$ & ষ্ঠ & ঔ & $\sum_{0}$ & $\sum_{O}$ & ঔ & 离 & \\
\hline 㟔 & క & 㟔 & ら & $\mathfrak{c}$ & $\grave{\omega}$ & క & 㟔 & 㟔 & 㟔 & 心 & \ & \\
\hline ? & 0 & 0 & 々 & Ш & 0 & U & U & U & 0 & U & 々 & \\
\hline 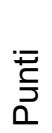 & $\begin{array}{l}\bar{\Phi} \\
\bar{U}\end{array}$ & $\begin{array}{l}\stackrel{\text { ఖ }}{\leftrightarrows} \\
\stackrel{0}{0}\end{array}$ & $\frac{\bar{\pi}}{\stackrel{\underline{\underline{T}}}{\frac{U}{U}}}$ & $\begin{array}{l}\frac{\pi}{\frac{\pi}{0}} \\
\frac{\pi}{0}\end{array}$ & $\begin{array}{l}\frac{\pi}{0} \\
\frac{\pi}{0} \\
\frac{\pi}{U}\end{array}$ & $\underset{\Xi}{\mathbb{\pi}}$ & $\frac{\mathbb{D}}{\mathbb{D}}$ & 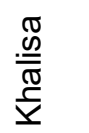 & 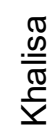 & & $\begin{array}{l}\frac{\pi}{\mathbb{N}} \\
\frac{\pi}{\infty} \\
\frac{\pi}{\infty}\end{array}$ & \\
\hline 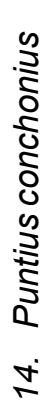 & 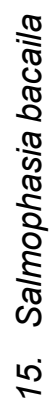 & 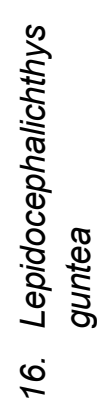 & 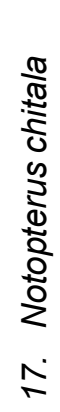 & $\begin{array}{l}\mathbb{D} \\
\frac{\mathbb{d}}{\mathbb{d}} \\
\frac{\pi}{\delta} \\
\frac{\mathbb{d}}{\mathbb{d}} \\
\infty \\
\infty\end{array}$ & 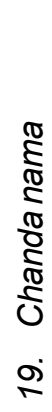 & 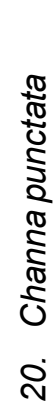 & 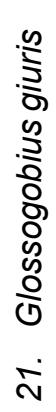 & 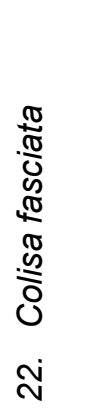 & $\begin{array}{l}\frac{\pi}{\pi} \\
\frac{\mathbb{d}}{0} \\
\dot{0} \\
0 \\
\stackrel{D}{0}\end{array}$ & 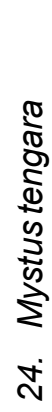 & 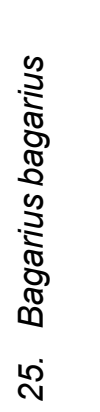 & $\begin{array}{l}\bar{\pi} \\
\overline{0}\end{array}$ \\
\hline & & $\begin{array}{l}\frac{\pi}{\pi} \\
\frac{\pi}{0} \\
: 0 \\
0 \\
0 \\
0\end{array}$ & $\begin{array}{l}0 \\
\frac{0}{\pi} \\
\frac{0}{2} \\
\frac{0}{2} \\
\frac{0}{0} \\
\frac{0}{2} \\
0\end{array}$ & $\begin{array}{l}0 \\
\mathbb{0} \\
\frac{\pi}{0} \\
\frac{0}{0} \\
\mathbb{D} \\
\mathbb{0} \\
\frac{\mathbb{Q}}{\varepsilon}\end{array}$ & & 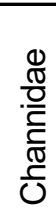 & $\begin{array}{l}0 \\
\frac{0}{0} \\
\frac{0}{0} \\
\overline{0} \\
0 \\
0\end{array}$ & 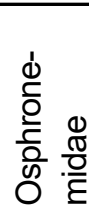 & & $\begin{array}{l}\frac{\mathbb{0}}{\pi} \\
\frac{0}{0} \\
\frac{0}{\pi} \\
\infty\end{array}$ & $\begin{array}{l}\frac{\pi}{\pi} \\
\frac{\pi}{0} \\
\frac{0}{0} \\
\frac{.0}{\omega}\end{array}$ & \\
\hline & & & 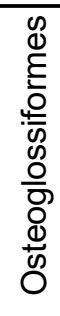 & 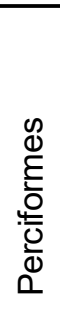 & & & & & & $\begin{array}{l}\stackrel{\mathscr{d}}{E} \\
\stackrel{\underline{0}}{\frac{2}{5}} \\
\stackrel{\bar{J}}{\bar{\omega}}\end{array}$ & & \\
\hline
\end{tabular}


TABLE-3 : 'Diversity index of fish assemblages among the five different sampling stations of Kangsabati River, Purulia district during the study period'.

\begin{tabular}{l|c|c|c|c|c}
\hline Site & $\begin{array}{c}\text { Species } \\
\text { Richness } \\
(\mathbf{D})\end{array}$ & $\begin{array}{c}\text { Shannon- } \\
\text { wiener index } \\
\left(\mathbf{H}^{\prime}\right)\end{array}$ & $\begin{array}{c}\text { Evenness } \\
\text { index } \\
(\mathbf{J})\end{array}$ & $\begin{array}{c}\text { Simpson's } \\
\text { dominance } \\
\text { index (D) }\end{array}$ & $\begin{array}{c}\text { Simpson's } \\
\text { diversity } \\
\text { index (1-D) }\end{array}$ \\
\hline $\mathbf{I}$ & 1.30 & 2.67 & 0.45 & 0.09 & 0.91 \\
\hline II & 1.31 & 2.65 & 0.45 & 0.09 & 0.91 \\
\hline III & 1.31 & 2.60 & 0.45 & 0.09 & 0.91 \\
\hline IV & 1.36 & 2.55 & 0.44 & 0.10 & 0.9 \\
\hline V & 1.40 & 2.52 & 0.44 & 0.10 & 0.9 \\
\hline Total & 0.61 & 2.61 & 0.35 & 0.09 & 0.91 \\
\hline
\end{tabular}

Temperature $(r=-0.861, p<0.05)$, level of Conductivity $(r=-0.881, p<0.05)$, Total Dissolved Solid and Water Temperature $(r=-0.878, p<0.05)^{16}$, the zooplankton density and Water Temperature $(r=-0.794, p<0.05)$, between the Species Richness of fishes and Conductivity $(r=-0.944, p<0.05)$, the Species Richness of fishes and Water Temperature $(r=-0.958, p<0.05)$. Negative correlations were also found between the Dissolved Oxygen and the Water Temperature $(r=-0.932)$. The Correlation between water parameters and biotic factor

TABLE-4 : 'Pearson's $r$ correlation matrix between abiotic and biotic parameters at Kangsabati River, Purulia district during the study period'.

\begin{tabular}{l|c|c|c|c|c|c|c|c|c}
\hline & WT & DO & PH & TRA & CON & TDS & SAL & Ш & SR \\
\hline WT & 1.000 & & & & & & & & \\
\hline DO & -0.932 & 1.000 & & & & & & & \\
\hline PH & -0.958 & 0.974 & 1.000 & & & & & & \\
\hline TRA & $-0.861^{*}$ & 0.986 & 0.935 & 1.000 & & & & & \\
\hline CON & 0.974 & -0.934 & -0.912 & $-0.881^{*}$ & 1.000 & & & & \\
\hline TDS & $-0.878^{*}$ & 0.985 & 0.921 & 0.992 & -0.916 & 1.000 & & & \\
\hline SAL & -0.984 & $0.941^{*}$ & 0.928 & $0.885^{*}$ & -0.998 & 0.916 & 1.000 & & \\
\hline D & $-0.794^{*}$ & $0.592^{*}$ & $0.747^{*}$ & 0.466 & $-0.649^{*}$ & 0.450 & $0.681^{*}$ & 1.000 & \\
\hline SR & $-0.958^{*}$ & 0.995 & 0.990 & 0.967 & $-0.944^{*}$ & 0.965 & 0.954 & $0.666^{*}$ & 1.000 \\
\hline
\end{tabular}

* represent the level of significance $0.05(p<0.05)$. 


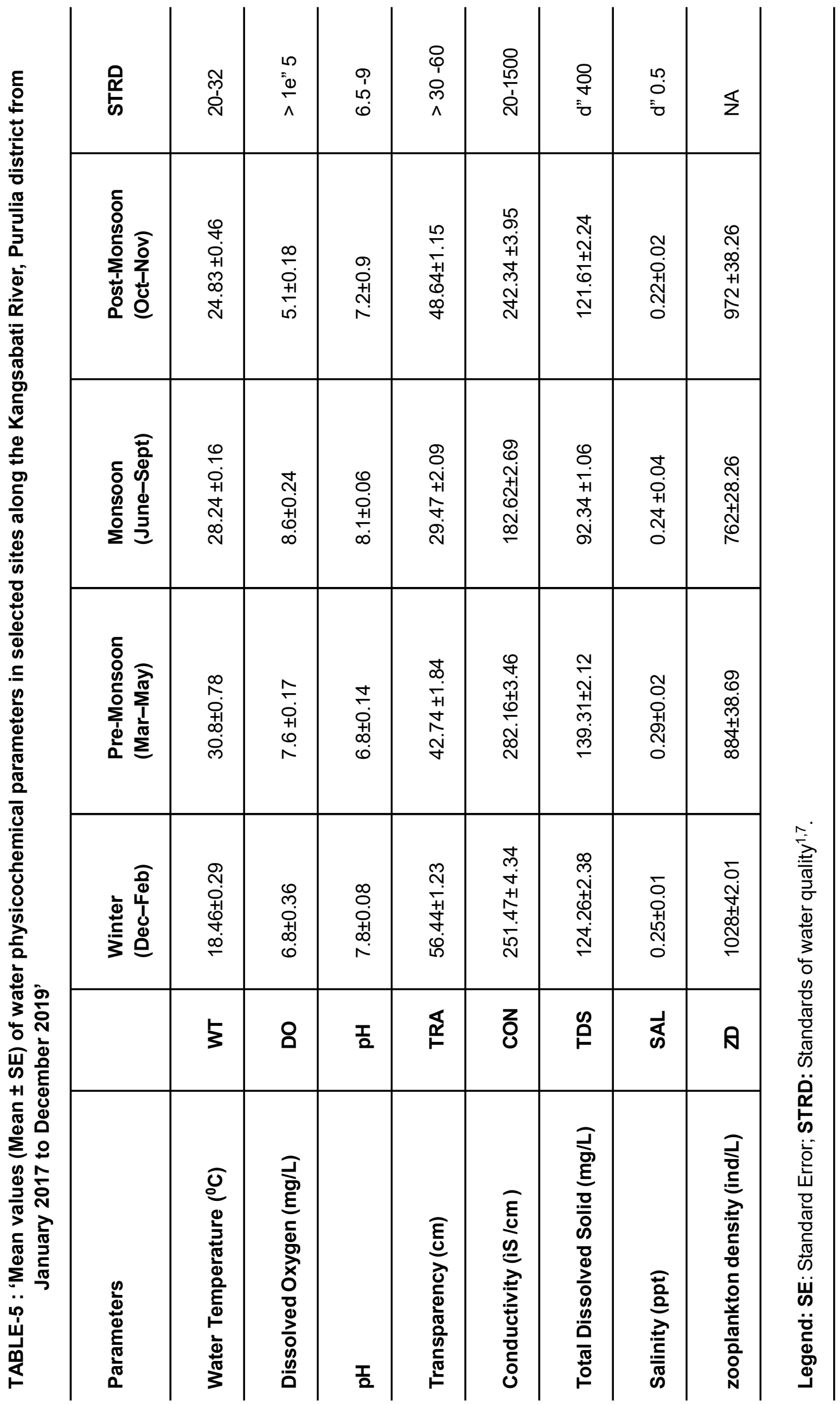




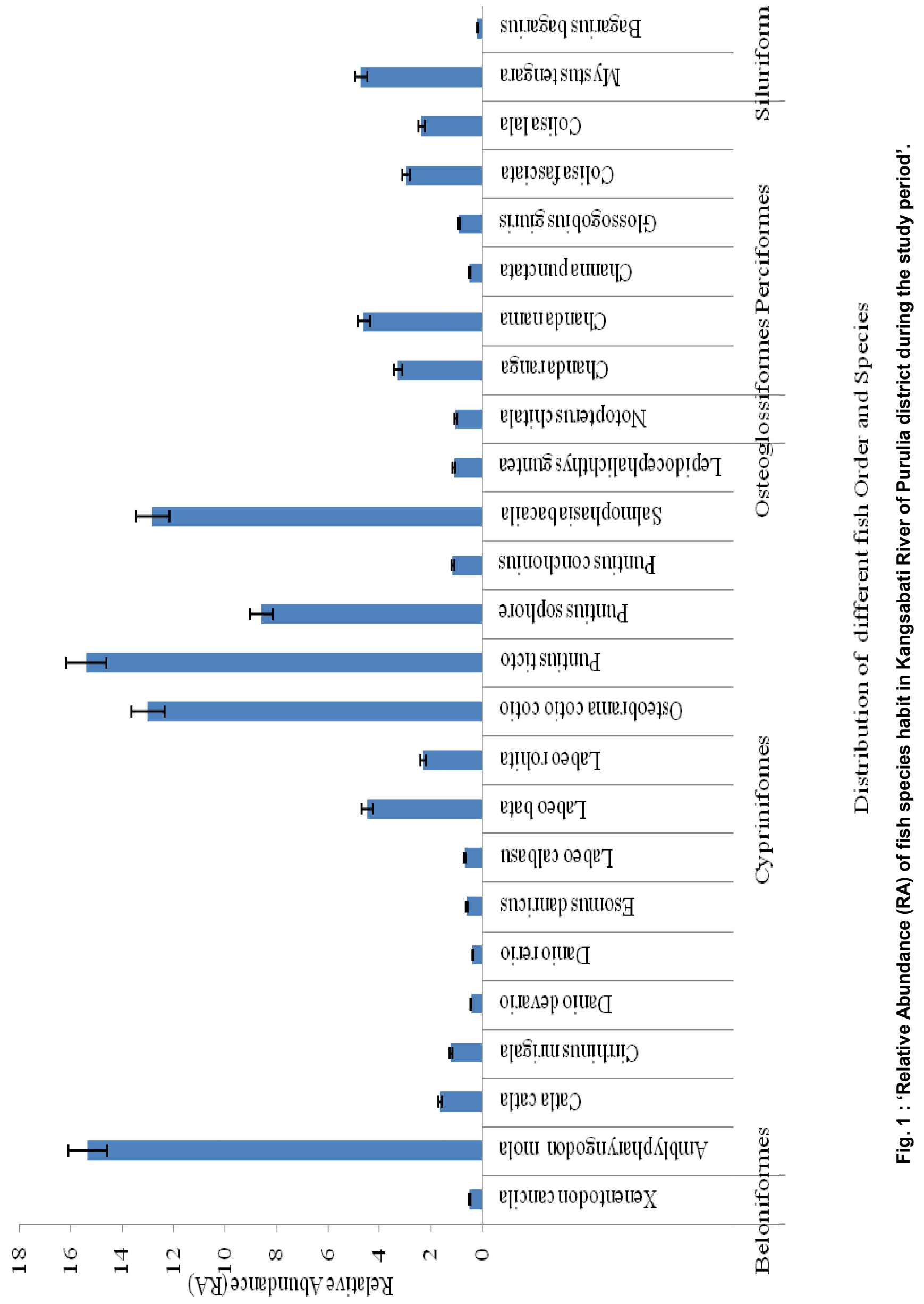


(zooplankton/fish population) during the study period shows at a glance in (Table-6).

\section{Discussion}

Studies of ecological diversity are essential to understand the species richness or taxonomic diversity, community structure, species assemblages in a given area, to understand the environmental conditions ${ }^{22}$. According to the observation, water quality shows the majority, of the components, were within the range. That can create a sustainable and favourable environment for the growth and propagation of icthyofaunal diversity. Although, the fish diversity and abundance was high in the upstream segment but progressive decline in the lower part of the Kangsabati River (Site IV: Pairachali bridge \& Site V: Darasol river bank). But according to the local fishermen 'two to three decades ago there was huge fish diversity in this area of the river. This arises a question, why it is so? The water of these two sites carried high quantity of Total Dissolved Solid and level of Conductivity compared to upstream (site I, II and III). The two sites accumulate runoff from upriver, which increases the sediment loads and organic matter from the nearby agricultural wastes, domestic pollutant, burning ghat effluents, dumped solid wastes, during monsoon and postmonsoon period, domestic sewage etc ${ }^{2,10}$. It was also noted that during the study period the extensive sand mining, siltation on the river bed has a strong negative impact on icthyofaunal diversity. So, the answer is Global climate change, pollution, soil erosion, overfishing and other anthropogenic activities are common factors and threats to fish biodiversity.

\section{Conclusion}

Fish is an economical source of protein and an important cash crop in West Bengal. Thus, the demands of fish cross the carrying capacity $(\mathrm{K})$ of the aquatic system. Subsequently, the lotic ecosystems are under constant pressure. According to the observation during the study period, the following steps are recommended for the restoration of declining fish diversity in the Kansabati River : (i) To control and manage the use of chemical fertilizers and harmful pesticides' in riverside agricultural activities (ii) water quality parameters should
TABLE-6: 'Correlation between water parameters and biotic factor (zooplankton/ fish population) during study period'.

\begin{tabular}{l|c|c|c}
\hline Parameters & & $\begin{array}{c}\text { Zoopla- } \\
\text { nkton }\end{array}$ & $\begin{array}{c}\text { Fish } \\
\text { population }\end{array}$ \\
\hline $\begin{array}{l}\text { Water } \\
\text { Temperature }\left({ }^{0} \mathrm{C}\right)\end{array}$ & WT & $-0.794^{*}$ & $-0.958^{*}$ \\
\hline $\begin{array}{l}\text { Dissolved Oxygen } \\
\text { (mg/L) }\end{array}$ & DO & $0.592^{*}$ & 0.995 \\
\hline $\mathrm{PH}^{\mathrm{H}}$ & PH & $0.747^{*}$ & 0.990 \\
\hline $\begin{array}{l}\text { Transparency } \\
\text { (cm) }\end{array}$ & TRA & 0.466 & 0.967 \\
\hline $\begin{array}{l}\text { Conductivity } \\
\text { (iS /cm ) }\end{array}$ & CON & $-0.649^{*}$ & $-0.944^{*}$ \\
\hline $\begin{array}{l}\text { Total Dissolved } \\
\text { Solid (mg/L) }\end{array}$ & TDS & 0.450 & 0.965 \\
\hline \begin{tabular}{l} 
Salinity (ppt) \\
\hline $\begin{array}{l}\text { zooplankton } \\
\text { density (ind/L) }\end{array}$
\end{tabular} & SAL & $0.681^{*}$ & 0.954 \\
\hline
\end{tabular}

be monitored regularly (iii) strictly prevent the deforestation surrounding the river bank (iv) Harvesting of fingerling/fry should stop (v) strictly ban the harvesting of mature female fishes in breeding seasons (vi) selective area (high anthropogenic stress) of the river should be declared as an aquatic sanctuary. Last but not the least, organize the maximum number of community awareness programs among the riverside common people for active participation in the conservation strategies and therefore ensure the sustainable ecological balance of the River. The work will provide a platform for the future researcher, to make a sustainable conservation plan for this region.

\section{References}

1. Ali M, Salam A, Azeem A, Shafique M, Khan BA. Studies on the effect of seasonal variations on physical and chemical characteristics of mixed water from Rivers Ravi and Chenab at union site in Pakistan. J. Res. BZ Univ. Multan. 2000; 2:1-7.

2. Arthington $\mathrm{AH}$, Dulvy NK, Gladstone W, Winfield IJ. Fish conservation in freshwater and marine realms: status, threats and management. Aquatic Conservation: Marine and Freshwater Ecosystems. 2016 Sep; 26(5):838-57.

3. Battish SK. Freshwater zooplankton of India Oxford \& IBH Publishing Co. Pvt Ltd., New Delhi, India. 1992. 
4. Bera A, Bhattacharya M, Patra BC, Sar UK. Ichthyofaunal diversity and water quality in the Kangsabati Reservoir, West Bengal, India. Advances in Zoology. 2014; 3(12):28-32.

5. Bhat A. Diversity and composition of freshwater fishes in river systems of Central Western Ghats, India. Environmental biology of fishes. 2003; 68(1):25-38.

6. Bonjoru R, Abubakar KA, Hassan El, Jerry TJ. Diversity and Abundance of Fish Species in Some Selected Riverine Wetlands of Upper Benue River Basin, Nigeria. Journal of Environmental Science, Toxicology and Food Technology. 2019; 13(8): 14-18.

7. Boyd CE, Tucker CS. "Ecology of aquaculture ponds." Pond aquaculture water quality management. Springer, Boston, MA, 1998. 8-86.

8. Das MK, Samanta S, Sajina AM, Sudheesan D, Naskar M, Bandopadhyay MK, Paul SK, Bhowmick S, Srivastava PK. Fish diversity, community structure and ecological integrity of river Brahmani. Journal of the Inland Fisheries Society of India. 2016; 48(1):1-3.

9. Eisenhauer N, Barnes AD, Cesarz S, Craven D, Ferlian O, Gottschall F, Hines J, Sendek A, Siebert J, Thakur MP, Türke M. Biodiversity-ecosystem function experiments reveal the mechanisms underlying the consequences of biodiversity change in real world ecosystems. Journal of Vegetation Science. 2016; 27(5):1061-7100.

10. Fu C, Wu J, Chen J, Wu Q, Lei G. Freshwater fish biodiversity in the Yangtze River basin of China: patterns, threats and conservation. Biodiversity \& Conservation. 2003; 12(8):1649-85.

11. Guo Q, Liu X, Ao X, Qin J, Wu X, Ouyang S. Fish diversity in the middle and lower reaches of the Ganjiang River of China: Threats and conservation. PloS one. 2018; 13(11):e0205116.

12. Hammer $\varnothing$, Harper DA, Ryan PD. PAST: Paleontological statistics software package for education and data analysis. Palaeontologia electronica. 2001; 4(1):9.

13. Heal GM, Barbier EB, Boyle KJ, Covich AP, Gloss SP, Hershner CH, Hoehn JP, Pringle CM, Polasky S, Segerson K, Shrader-Frechette K.. National Research Council. Valuing ecosystem services: toward better environmental decision-making. National academies Press; 2005 May 14.

14. https://www.analystsoft.com/en/

15. https://www.google.com/earth/

16. Hu B, Hu X, Nie X, Zhang X, Wu N, Hong Y, Qin HM. Seasonal and inter-annual community structure characteristics of zooplankton driven by water environment factors in a sub-lake of Lake Poyang, China. PeerJ. 2019; 7:e7590.

17. IUCN (International Union for Conservation of Nature and Natural Resources) (2008) Available at www.iucnredlist.org

18. Jayaram KC. The freshwater fishes of the Indian region. Narendra Publishing House, New Delhi; 1999.

19. Jhingran VG. Fish and Fisheries of India, Hindustan Publ. Co., New Delhi; 1991.

20. Kar A, Bhattacharya M, Ghorai M, Patra S, Patra BC. Ichthyofaunal Diversity of Kangsabati River at Paschim Medinipur District, West Bengal, India. In Proceedings of the zoological society, Springer India.2017; 70(2):165173.

21. Manjare SA, Vhanalakar SA, Muley DV. Analysis of water quality using physicochemical parameters Tamdalge tank in Kolhapur district, Maharashtra. International Journal of Advanced Biotechnology and Research. 2010; 1(2):115-119.

22. Negi RK, Mamgain S. Species diversity, abundance and distribution of fish community and conservation status of Tons River of Uttarakhand State, India. Journal of Fisheries and Aquatic Science. 2013; 8(5):617-26.

23. Siligato S, Böhmer J. Using indicators of fish health at multiple levels of biological organization to assess effects of stream pollution in southwest Germany. Journal of Aquatic Ecosystem Stress and Recovery. 2001; 8(34):371-86.

24. Suresh, V. R., Sahu, S. K., Manna, S. K., Roy, S., Karnatak, G., Ekka, A., and Chandra, G. ICAR-Central Inland Fisheries Research Institute Barrackpore, Kolkata-700 120, West Bengal, Annual report 2015-16. 2016.

25. Talwar PK. Inland fishes of India and adjacent countries. CRC Press; 1991.

26. Yuan Y, Jiang M, Liu X, Yu H, Otte ML, Ma C, Her YG. Environmental variables influencing phytoplankton communities in hydrologically connected aquatic habitats in the Lake Xingkai basin. Ecological Indicators. 2018; 91:1-2. 